\title{
Is mitochondrial DNA turnover slower than commonly assumed?
}

\section{Other Journal Item}

\section{Author(s):}

Poovathingal, Suresh Kumar; Gruber, Jan; Lakshmanan, LakshmiNarayanan; Halliwell, Barry; Gunawan, Rudiyanto

Publication date:

2012-10

Permanent link:

https://doi.org/10.3929/ethz-b-000056773

Rights / license:

In Copyright - Non-Commercial Use Permitted

Originally published in:

Biogerontology 13(5), https://doi.org/10.1007/s10522-012-9390-7 


\title{
Is mitochondrial DNA turnover slower than commonly assumed?
}

\author{
Suresh Kumar Poovathingal · Jan Gruber • \\ LakshmiNarayanan Lakshmanan • \\ Barry Halliwell · Rudiyanto Gunawan
}

Received: 7 March 2012/ Accepted: 21 July 2012/Published online: 3 August 2012

(C) Springer Science+Business Media B.V. 2012

\begin{abstract}
Mutations arise during DNA replication due to oxidative lesions and intrinsic polymerase errors. Mitochondrial DNA (mtDNA) mutation rate is therefore closely linked to the mitochondrial DNA turnover process, especially in post mitotic cells. This makes the mitochondrial DNA turnover rate critical for understanding the origin and dynamics of mtDNA mutagenesis in post mitotic cells. Experimental mitochondrial turnover quantification has been based on different mitochondrial macromolecules, such as mitochondrial proteins, lipids and DNA, and the experimental data suggested highly divergent turnover rates, ranging from over 2 days to about 1 year. In this article we argue
\end{abstract}

Suresh Kumar Poovathingal and Jan Gruber have equally contributed to this study.

Electronic supplementary material The online version of this article (doi:10.1007/s10522-012-9390-7) contains supplementary material, which is available to authorized users.

\section{S. K. Poovathingal}

Luxembourg Centre for Systems Biomedicine (LCSB), University of Luxembourg, Campus Belval, 7, avenue des Hauts-Fourneaux, 4362 Esch-sur-Alzette, Luxembourg e-mail: suresh.poovathingal@uni.lu

J. Gruber · B. Halliwell

Department of Biochemistry, Neurobiology and Ageing

Program, Centre for Life Science (CeLS),

28 Medical Drive, Singapore 117456, Singapore

e-mail: jan_gruber@nus.edu.sg

B. Halliwell

e-mail: bchbh@nus.edu.sg that mtDNA turnover rate cannot be as fast as is often envisaged. Using a stochastic model based on the chemical master equation, we show that a turnover rate corresponding to mtDNA half-life in the order of months is the most consistent with published mtDNA mutation levels.

Keywords Mitochondrial DNA - Turnover rate · Ageing · Point mutations · POLG transgenic mice

\section{Mitochondrial and mitochondrial DNA turnover}

Accumulation of mitochondrial DNA (mtDNA) mutations and mitochondrial dysfunction are often suggested to be important mechanisms of ageing (Harman 1972). Since mutations predominantly occur during

\footnotetext{
L. Lakshmanan

Department of Chemical \& Biomolecular Engineering, National University of Singapore, 4 Engineering Drive 4, Blk E5 \#02-09, Singapore 117576, Singapore

e-mail: lakshminarayanan@nus.edu.sg

R. Gunawan $(\bowtie)$

Institute for Chemical and Bioengineering, ETH Zurich, HCI F 101, Wolfgang-Pauli-Strasse 10, 8093 Zurich, Switzerland e-mail: rudi.gunawan@chem.ethz.ch
} 
mtDNA replication, either as a consequence of oxidative lesions or of intrinsic polymerase errors (Kujoth et al. 2007), mtDNA turnover rate is a critical parameter determining de novo mtDNA mutation rate (Poovathingal et al. 2009). The mtDNA turnover rate may also impact clonal expansion dynamics of mtDNA mutations (Chinnery and Samuels 1999; Nicholas et al. 2009; Poovathingal et al. 2009). In post-mitotic tissues, mtDNA turnover is often considered to be mainly associated with the biogenesis of mitochondria that balances the mitochondrial autophagy (mitophagy) process, where the complete organelle and its constituents are catabolized by cellular double membrane structures known as autophagosomes (Nakatogawa et al. 2009). However, mitochondria do not generally exist as disconnected organelles, but undergo rapid fusion and fission, forming large, complex reticular structures (Chen and Chan 2005). Therefore, it is not trivial to define what exactly constitutes the "turnover of a mitochondrion". Despite of this, mitochondrial turnover rates have been proposed based on the turnover rates of various mitochondrial constituents such as mitochondrial proteins (Lipsky and Pedersen 1981; Menzies and Gold 1971; Miwa et al. 2008), mtDNA (Collins et al. 2003; Gross et al. 1969; Korr et al. 1998) or lipids (cardiolipin) (Fletcher and Sanadi 1961; Gross et al. 1969; Huemer et al. 1971).

Experimental data on the turnover of mitochondrial components are limited and divergent. Reported halflives range from $\sim 2$ to 350 days, depending on reference molecules and measurement techniques (Collins et al. 2003; Gross et al. 1969; Huemer et al. 1971; Korr et al. 1998; Miwa et al. 2008). Half-life values of about 2-17 days, derived from bulk protein turnover data, are often cited as the global mitochondrial turnover rate (Fletcher and Sanadi 1961; Menzies and Gold 1971; Miwa et al. 2008). However, as argued above, it is unclear if a global mitochondrial turnover can in fact be meaningfully defined. Importantly, if these short half-life values are assumed to be indicative of the mtDNA turnover rate, they would have dramatic impact on age-dependent mtDNA mutation accumulation dynamics (Chinnery and Samuels 1999; Elson et al. 2001; Kowald et al. 2005; Kowald and Kirkwood 2000; Poovathingal et al. 2009). It is the latter relationship that we will explore in more detail below.

\section{Challenges with estimating global mitochondrial turnover rate-implications in estimating mtDNA turnover rate}

Mitochondrial protein turnover rates are commonly used as a proxy for the turnover of the complete mitochondrial organelle (Fletcher and Sanadi 1961; Miwa et al. 2008) and thus for mtDNA turnover. Mitochondrial protein turnover rates are usually measured using pulse chase experiments (Fletcher and Sanadi 1961; Menzies and Gold 1971; Miwa et al. 2008). In these experiments, isotope-labeled protein precursor molecules are administered, followed by tracking the labeled mitochondrial protein. The firstorder decay rate for the loss of isotope-label yields mitochondrial protein half-lives. However, such data must be interpreted with caution when extending this to estimate turnover rate of other mitochondrial components. For one, mitochondria contain $\sim 1,200$ different polypeptides (Mootha et al. 2003), with reported half-lives ranging from a little over an hour to more than 10 days (Table 1). The presence of intramitochondrial proteolytic systems (Mijaljica et al. 2007) implies that mitochondrial proteins can be degraded independent of organelle turnover, resulting in protein-specific half-lives (Jaleel et al. 2008). In contrast to proteomics approaches, pulse chase experiments utilizing bulk protein will thus result in halflives that reflect the average turnover of the population of proteins in mitochondria (Miwa et al. 2008). Therefore, protein turnover data from such experiments may be dominated by the turnover rate of few abundant proteins. This suggests that, even with the best experimental practices and even when artifacts, such as precursor reutilization (Burgess et al. 1978; Lipsky and Pedersen 1981; Miwa et al. 2008), are carefully controlled, bulk protein turnover might still overestimate the turnover of mitochondrial components, such as mtDNA.

Individual mitochondrial protein half-lives of more than 10 days have been reported (Table 1). If these experimental data are accepted, mitochondrial halflife, if meaningful at all, cannot be shorter than 10 days. However, more data from mitochondrial proteomics studies using modern methods would be desirable [e.g.:(Dai et al. 2009)]. Long half-lives have also been reported for other macromolecules in mitochondria. For instance, a half-life of 40 days has 
Table 1 Turnover rates of some mammalian mitochondrial proteins

\begin{tabular}{lllll}
\hline S. no. & Protein & Half life & Organism/tissue & Reference \\
\hline 1 & Ornithine aminotransferase & 1.9 days & Rat liver & Ip et al. (1974) \\
2 & Cytochrome oxidase & 5.7 & Rat liver & Ip et al. (1974) \\
3 & Carbamyl phosphate synthetase & 7.7 & Rat & Nicoletti et al. (1977) \\
4 & Malate dehydrogenase & 2.6 & Rat & Nicoletti et al. (1977) \\
5 & Aminolevulinate synthetase & 70 min & Rat liver & Marver et al. (1966) \\
6 & Cytochrome c & 10.3 days & Rat liver & Fletcher and Sanadi (1961) \\
7 & Ornithine transcarbamylase & 7.7 days & Rat liver & Wallace et al. (1986) \\
8 & Cytochrome c & 11.8 days & Rabbit heart & Grozdova and Starostina (1973) \\
9 & 66 different mitochondrial proteins & $2-8.5$ days & Rat skeletal muscle & Jaleel et al. (2008) \\
10 & Various & $\sim 18-95$ days & Rat brain & Rodrijguez de Lores et al. (1971) \\
\hline
\end{tabular}

been reported for the mitochondrial lipid cardiolipin (Huemer et al. 1971). In this work, we are mainly concerned with the turnover of mtDNA. Importantly, attempts at direct measurement of mtDNA turnover have yielded half-life values ranging from 10 days up to as high as 300 days, again depending on tissue types and experimental techniques (Collins et al. 2003; Gross et al. 1969; Korr et al. 1998). However, even the fastest reported mtDNA turnover rate (10 days) (Gross et al. 1969) is significantly longer than the two-day mitochondrial turnover rate estimated based on the bulk protein measurements (Miwa et al. 2008).

\section{Physiological levels of mtDNA turnover rate- mtDNA mutation data}

The turnover rate of mtDNA critically determines how fast de novo mtDNA mutations can occur, as each replication event has a finite probability of introducing a new mutation (Poovathingal et al. 2009). For the purpose of this study, we considered age-related mtDNA point mutations in a TaqI recognition site (bp 634-637) in mouse heart tissues as reported by Vermulst et al. (2007). The majority of these mutations ( $86 \%$ ) are GC to AT transitions and for the TaqI site investigated by Vermulst et al. (2007), such mutations are silent, causing no change in amino acid sequence of the relevant gene product and therefore cannot be subjected to selective degradation. Furthermore, even for those mutations that are not silent, the reported burdens are several orders of magnitude lower than the levels that have been shown to affect the electron transport chain (ETC). function (Inoue et al. 2000; Vermulst et al. 2007). If these low levels of mtDNA mutation burden are considered to be true, then these data provide a constraint on the magnitude of the mtDNA turnover rate, because ultimately mtDNA turnover directly affects the observed de novo mtDNA point mutation rate.

Using in silico simulations of random mtDNA turnover and de novo mutations, we argue that mtDNA turnover cannot be as rapid as is often envisaged based on the mitochondrial protein turnover data. A stochastic model based on chemical master equation (CME) (Poovathingal et al. 2009) was used to simulate the mitochondrial DNA turnover process and mtDNA mutagenesis process at different mtDNA turnover rates in cells. Using this stochastic model, we have simulated the accumulation of mtDNA point mutations burden in mouse heart tissue for several reported mitochondrial turnover rates and then compared the simulated burdens against the reported data.

In an earlier work (Poovathingal et al. 2009), we have demonstrated that the faster turnover promotes more rapid accumulation of mtDNA mutations. Here this model has been used to investigate the constraint on possible mtDNA turnover rate, given the observed mtDNA point mutation burden. We apply this model to the cases of wild-type and polymerase- $\gamma$ (POLG) mutator mice, for which mtDNA point mutation data have been previously reported (Vermulst et al. 2007). The in silico mtDNA model tracks the accumulation of point mutations in cardiomyocytes $\left(2.5 \times 10^{7}\right.$ cells $)$ during two stages of life: development and postdevelopment (Fig. S1 in Online Resource 1). In the model, we assumed that the mtDNA population in each cell consists of only wild-type $(W)$ and mutant 
$(M)$ molecules and that these mtDNA are well-mixed due to fast fusion-fission (Chinnery and Samuels 1999; Poovathingal et al. 2009). The stochastic model was formulated as a CME that describes the random mtDNA turnover process, comprising of relaxed replication and degradation of mtDNA (details in the Online Resource 1). Importantly, mutant and wild-type mtDNA are equally likely to replicated or degraded during mtDNA turnover. De novo point mutations are assumed to result from mtDNA replication errors due to two cumulative factors: oxidative lesions associated with ROS and the finite fidelity of the mtDNA polymerase- $\gamma$ (POLG) enzyme (Kunkel 1992; Zhang et al. 2000) (Fig. S2 in Online Resource 1).

Oxidative lesions in mtDNA, including but not limited to $8 \mathrm{OHdG}$, one of the oxidation products of guanine, facilitate the formation of point mutations during mtDNA replication because of alternative hydrogen bonding configuration of the oxidized bases leading to inappropriate base-paring. Therefore, the presence of oxidative lesions such as $8 \mathrm{OHdG}$ during mtDNA replication is an important determinant of mutation probability during replication. Essentially, mtDNA replication can turn oxidative lesions in the template strand (oxidative damage) into point mutations in the newly synthesized strand (Halliwell and Aruoma 1991; Halliwell and Gutteridge 1999). It is extremely challenging to accurately determine the rate of de novo oxidative damage events and of repair of such damage in vivo. The most widely available parameter related to oxidative DNA damage is therefore the steady state damage level of various oxidative DNA lesions, including 8OHdG. Steady state damage level results from the balance between the damage rate and repair rate. At any given time, there is a small fraction of the total damage events that remain unrepaired. Such steady state damage levels are typically very low, representing only a tiny fraction of the damage events that occur.

In vivo $8 \mathrm{OHdG}$ burden in nuclear DNA has been reported to be between 0.3 and 4.2 lesions per $10^{6} \mathrm{bp}$ based on different samples and different measurement techniques (ESCODD 2002a, b). However, such lesions make up only about $10-20 \%$ of the complete damage spectra (Halliwell and Aruoma 1991; Halliwell and Gutteridge 1999) and thus, the overall oxidative lesions could range between 1.5 and 4.2 lesions per $10^{6} \mathrm{bp}$. In our model simulations, we have made a conservative assumption that the oxidative damage to mtDNA is the same as that to nuclear DNA and have also used the lowest value in the range $\left(1.5 \times 10^{-6} \mathrm{bp}^{-1}\right)$. This means that our model simulations likely underestimate the overall mutation rate during mtDNA replication. Importantly, oxidative lesions are converted into mutations only when mtDNA is replicated.

On the other hand, the reported POLG fidelity values range from $10^{-7}$ to $10^{-4}$ mutations $\mathrm{bp}^{-1}$ replication $^{-1}$, depending on cell types and quantification assays (Johnson and Johnson 2001; Kunkel 1985; Kunkel et al. 1987; Lee and Johnson 2006). In the simulations of the POLG transgenic mice, the replication error frequency due to POLG fidelity was set to 200 times higher than that of the wild-type mouse, based on the commonly reported literature data (Kunkel 1992; Zhang et al. 2000). Again, we have used the most conservative (lowest) values from the above range when computing the frequency of mtDNA replication error. Thus the mutation burden obtained by our model simulations should represent a lower limit (more details in Online Resource 1).

Many mitochondrial related processes were left out from the model, such as selective degradation of mitochondria with lower membrane potential (possibly due to mitochondria harboring high levels of detrimental mtDNA mutations) (Kim et al. 2007) and retrograde signaling (Liu and Butow 2006). While each of these processes may complicate or mask the inference of mtDNA turnover rate, we note that such processes are relevant only when mtDNA mutations become physically detectable, for instance through effects on the ETC activity, ATP or ROS levels or through impact on mitochondrial membrane potential (Kanki and Klionsky 2008; Narendra et al. 2008; Twig et al. 2008). Obviously, for highly deleterious mtDNA mutations, selective degradation of the affected mitochondria could serve as their clearance mechanism and by ignoring this process, our model simulation analysis may suggest an artificially slow mtDNA turnover rate. On the other hand, if retrograde signaling was prominent, the mtDNA turnover rate predicted by our basic model would be an exaggeration.

Mutant mtDNA carrying a single point mutation is indistinguishable from WT DNA unless the mutation causes some sort of phenotypes. Meanwhile, the mechanisms proposed for selective degradation and retrograde signaling assume functionally relevant 
mutations (that is, mutations causing mitochondrial deficiency). This means that, while it is possible that such mechanisms operate to control the accumulation of some detrimental mutations such as mtDNA deletions or pathological point mutations, they cannot impact the accumulation of mutations that have no functional effect on mitochondria. In particular, selective degradation and retrograde signaling cannot impact the accumulation of silent point mutations. The stochastic drift of these mutations should arise only from mtDNA turnover, which was captured in our basic model as stated above. Such a simple model has other benefits, for example only a few model parameters need to be specified. In this case, the model involves only two free parameters, which are the mtDNA copy number at steady state and the de novo mutation frequency. The remaining model parameter is associated with the half-life of mtDNA. Therefore, model simulations reflected a direct causal relationship between mtDNA turnover and mutation burden.

Simulations of the model in this work were performed to predict the accumulation of mtDNA point mutations in post mitotic heart tissue of wildtype and POLG mutator mice. The steady state mtDNA copy number was set based on the reported value for mouse heart tissue (see supporting information in Online Resource 1), from which the mtDNA mutation burden were measured (Vermulst et al. 2007). Finally, model simulations were carried out using modified Gillespie's algorithm (see Online Resource 1, online for more details) and the complete model parameters used are included in the Online Resource 2, 3 and 4.

We have compared the mtDNA point mutation burden data from wild-type (WT) and POLG mutator mice (Vermulst et al. 2007) with those predicted by model simulations above with different mtDNA turnover rates, as shown in Fig. 1. The modeling results clearly show that, as long as neither mutant nor wild-type mtDNA are preferentially degraded or replicated, higher turnover rates always result in increased mtDNA mutation burden. This is not surprising, since de novo mtDNA mutations are tied to mtDNA replication. Note that we have explicitly made conservative assumptions regarding the occurrence of de novo mutations in this model, by setting the error rate arising due to limited POLG fidelity and lesions arising due to oxidative damage to the lowest plausible values that are consistent with the literature.
Figure 1d shows that the mtDNA mutation burden obtained using a short mitochondrial half-life [ $\sim 2$ days (Miwa et al. 2008)] does not fit well with the point mutation burden experimental data (Vermulst et al. 2007). Simulations using other reported half-lives, ranging from 10 to 300 days (Collins et al. 2003; Gross et al. 1969; Korr et al. 1998; Menzies and Gold 1971) as shown in Fig. 1a-c, indicate that the observed mtDNA mutation burden data for both the WT and POLG mice are most consistent with a mtDNA turnover rate corresponding to a half-life between 30 and 300 days. However, as we have taken a conservative assumption regarding the frequency of de novo mutations in this model, the actual mtDNA turnover rate could even be slower.

A slow mtDNA turnover rate during aging implies that de novo somatic mtDNA mutations are rare events. As we have discussed elsewhere (Poovathingal et al. 2009), for 300 day mtDNA half-life, the number of de novo mutations during the developmental stage of mice (where mtDNA replication frequency is tied to cell divisions) is roughly equal to that during the entire adult life of the organisms. In simulations, each somatic cell experiences on average between 2 and 3 de novo mutations during 3 years of mouse lifespan. Therefore, most mtDNA mutations may already exist in early life, consistent with findings from a deep sequencing study (Ameur et al. 2011). Also, these single mutational events would become the starting point for the (random) accumulation of mtDNA mutations during aging, a process known clonal expansion (Larsson 2011). An increase in either mtDNA turnover rate or replication error rate (e.g. due to increased oxidative lesions) would mean a higher de novo mtDNA mutation frequency. A more detailed exposition on the relationship between mtDNA turnover and age-related mtDNA mutation dynamics has also been given in an earlier work (Poovathingal et al. 2009).

\section{Concluding remarks}

Attributing a global mitochondrial half-life parameter based on the turnover of any individual mitochondrial components requires strong justification and given the dynamic characteristics of mitochondria, the very concept of "whole organelle" turnover may be questionable. We believe that there are strong arguments for mtDNA half-lives to be longer than a month. 

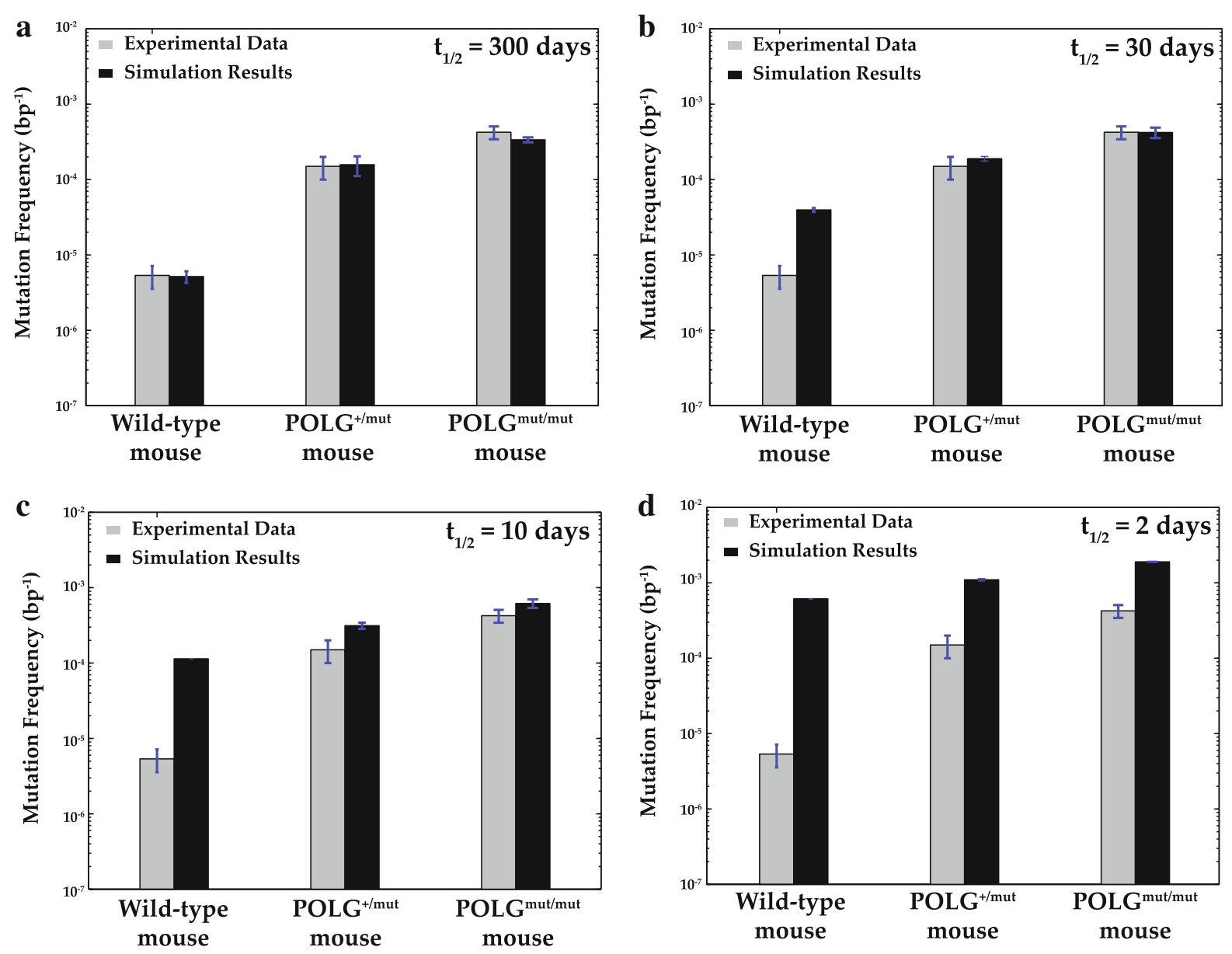

Fig. 1 Comparison of experimental data and simulations of point mutation frequency in wild-type and POLG mice $(n=10)$ (Vermulst et al. 2007) for different values of mtDNA turnover rate. The frequencies of the accumulated point mutations in mouse heart at the end of 29 months for wild-type mouse and at the end of 6 months of age for POLG ${ }^{+/ m u t}$ and POLG ${ }^{\text {mut } / m u t}$

If a global organelle turnover parameter were to be at all meaningful, it would have to be much slower than commonly quoted mitochondrial turnover time of 2-10 days (Gross et al. 1969; Miwa et al. 2008). Interestingly, there are several studies reporting longer half-lives ( $>30$ days) for cardiolipin, mtDNA and some individual proteins. As shown here, support for slow turnover of mtDNA comes from the quantification of age-related mtDNA mutations. Using a stochastic computational model based on minimal and conservative assumptions regarding the mtDNA turnover process and the associated de novo mutagenesis, we found that simulations using a short mtDNA half-life of 2 days grossly overestimate

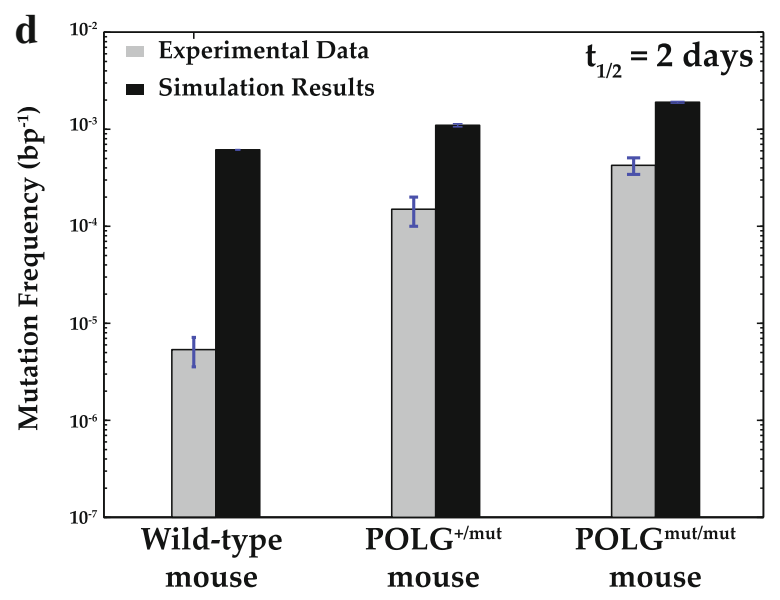

mice. Please note that the mutation frequency coordinate is in $\log$-scale. Results of point mutation frequencies are obtained using mtDNA turnover rates of a. $t_{1 / 2}=2$ days, (Miwa et al. 2008) b. $t_{1 / 2}=10$ days, (Gross et al. 1969) c. $t_{1 / 2}=30$ days, (Gross et al. 1969) and d. $t_{1 / 2}=300$ days (Collins et al. 2003)

mtDNA point mutations when compared with published data for mutation burden (Vermulst et al. 2007). The available experimental data were most consistent with a mtDNA half-life of months. In parallel to our conclusion, a previous computational study suggested that mitochondrial deoxyribonucleoside salvage pathway alone could not support a rapid mtDNA replication $(\sim 2 \mathrm{~h}$ mtDNA) and that the kinetics of this pathway could only provide for a much slower mtDNA replication of longer than $10 \mathrm{~h}$ (Gandhi and Samuels 2011).

The rate of mtDNA turnover is a crucial parameter, determining the rates of de novo mutations and the consequent age-dependent loss of mtDNA integrity 
and mitochondrial function (Gruber et al. 2008; Poovathingal et al. 2009; Wiesner et al. 2006). Given the fundamental role of mtDNA turnover rate for the understanding of the basic mechanism(s) of ageing, we feel that the accurate quantification of this rate is an important issue worthy of further careful experimental studies, if possible, by a direct and sensitive experimental estimation of mtDNA turnover. While some components of mitochondria are turned over rapidly, the global mitochondrial turnover rate, if meaningful at all, could not be faster than that of the most persistent macromolecules, e.g. mtDNA. Certainly, bulk protein turnover rate should not be used as a surrogate for mtDNA turnover.

Acknowledgments This work was supported by the Singapore Ministry of Education Grants [AcRF Tier 1, FRC Grant (R279000299112 to SKP and RG) and AcRF Tier 2 (MOE2010T2-2-048 to SKP, JG, and BH)], Singapore and Biomedical Research Council of Singapore [Grant number: (BMRC 07/1/ 21/19/524 to SKP, JG and BH)], and ETH Zurich (to RG and LL).

\section{References}

Ameur A, Stewart JB, Freyer C, Hagstrom E, Ingman M, Larsson NG, Gyllensten U (2011) Ultra-deep sequencing of mouse mitochondrial DNA: mutational patterns and their origins. PLoS Genet 7(3):e1002028

Burgess RJ, Walker JH, Mayer RJ (1978) Choice of precursors for the measurement of protein turnover by the doubleisotope method. Application to the study of mitochondrial proteins. Biochem J 176(3):919-926

Chen H, Chan DC (2005) Emerging functions of mammalian mitochondrial fusion and fission. Hum Mol Genet 14(2): R283-R289

Chinnery PF, Samuels DC (1999) Relaxed replication of mtDNA: a model with implications for the expression of disease. Am J Hum Genet 64:1158-1165

Collins ML, Eng S, Hoh R, Hellerstein MK (2003) Measurement of mitochondrial DNA synthesis in vivo using a stable isotopemass spectrometric technique. J Appl Physiol 94(6): 2203-2211

Dai DF, Santana LF, Vermulst M, Tomazela DM, Emond MJ, MacCoss MJ, Gollahon K, Martin GM, Loeb LA, Ladiges WC, Rabinovitch PS (2009) Overexpression of catalase targeted to mitochondria attenuates murine cardiac aging. Circulation 119(21):2789-2797

de Rodrijguez Lores A, de Alberici Canal M, de Robertis E (1971) Turnover of proteins in subcellular fractions of rat cerebral cortex. Brain Res 31(1):179-184

Elson JL, Samuels DC, Turnbull DM, Chinnery PF (2001) Random intracellular drift explains the clonal expansion of mitochondrial DNA mutations with age. Am J Hum Genet 68:802-806
ESCODD (2002a) Comparative analysis of baseline 8-oxo-7,8dihydroguanine in mammalian cell DNA, by different methods in different laboratories: an approach to consensus. Free Radic Res 23:2129-2133

ESCODD (2002b) Inter-laboratory validation of procedures for measuring 8-oxo-7,8-dihydroguanine/8-oxo-7,8-dihydro$2^{\prime}$-deoxyguanosine in DNA. Free Radic Res 36(3):239-245

Fletcher MJ, Sanadi DR (1961) Turnover of rat-liver mitochondria. Biochim Biophys Acta 51:356-360

Gandhi VV, Samuels DC (2011) Enzyme kinetics of the mitochondrial deoxyribonucleoside salvage pathway are not sufficient to support rapid mtDNA replication. PLoS Comput Biol 7(8):e1002078

Gross NJ, Getz GS, Rabinowitz M (1969) Apparent turnover of mitochondrial deoxyribonucleic acid and mitochondrial phospholipids in the tissues of the rat. J Biol Chem 244(6): 1552-1562

Grozdova MD, Starostina IK (1973) Protein synthesis in the myocardium in experimental allergic lesion of the heart in rabbits. Vopr Med Khim 19(4):403-407

Gruber J, Schaffer S, Halliwell B (2008) The mitochondrial free radical theory of ageing - where do we stand? Front Biosci 13:6554-6579

Halliwell B, Aruoma OI (1991) DNA damage by oxygenderived species. Its mechanism and measurement in mammalian systems. FEBS Lett 281(1-2):9-19

Halliwell B, Gutteridge JMC (1999) Free radicals in biology and medicine, 3rd edn. Oxford science publications, Clarendon Press, Oxford University Press, Oxford

Harman D (1972) The biologic clock: the mitochondria? J Am Geriatr Soc 20(4):145-147

Huemer RP, Lee KD, Reeves AE, Bickert C (1971) Mitochondrial studies in senescent mice-II. Specific activity, buoyant density, and turnover of mitochondrial DNA. Exp Gerontol 6(5):327-334

Inoue K, Nakada K, Ogura A, Isobe K, Goto Y, Nonaka I, Hayashi JI (2000) Generation of mice with mitochondrial dysfunction by introducing mouse mtDNA carrying a deletion into zygotes. Nat Genet 26(2):176-181

Ip MM, Chee PY, Swick RW (1974) Turnover of hepatic mitochondrial ornithine aminotransferase and cytochrome oxidase using [14C]carbonate as tracer. Biochim Biophys Acta 354(1):29-38

Jaleel A, Short KR, Asmann YW, Klaus KA, Morse DM, Ford GC, Nair KS (2008) In vivo measurement of synthesis rate of individual skeletal muscle mitochondrial proteins. Am J Physiol Endocrin ol Metab 295(5):E1255-E1268

Johnson AA, Johnson KA (2001) Fidelity of nucleotide incorporation by human mitochondrial DNA polymerase. J Biol Chem 276(41):38090-38096

Kanki T, Klionsky DJ (2008) Mitophagy in yeast occurs through a selective mechanism. J Biol Chem 283(47):32386-32393

Kim I, Rodriguez-Enriquez S, Lemasters JJ (2007) Selective degradation of mitochondria by mitophagy. Arch Biochem Biophys 462(2):245-253

Korr H, Kurz C, Seidler TO, Sommer D, Schmitz C (1998) Mitochondrial DNA synthesis studied autoradiographically in various cell types in vivo. Braz J Med Biol Res 31(2):289-298

Kowald A, Kirkwood TBL (2000) Accumulation of defective mitochondria through delayed degradation of damaged 
organelles and its possible role in the ageing of post-mitotic and dividing cells. J Theor Biol 202:145-160

Kowald A, Jendrach M, Pohl S, Bereiter-Hahn J, Hammerstein P (2005) On the relevance of mitochondrial fusions for the accumulation of mitochondrial deletion mutants: a modelling study. Aging Cell 4(5):273-283

Kujoth GC, Bradshaw PC, Haroon S, Prolla TA (2007) The role of mitochondrial DNA mutations in mammalian aging. PLoS Genet 3(2):e24

Kunkel TA (1985) The mutational specificity of DNA polymerases-alpha and-gamma during in vitro DNA synthesis. J Biol Chem 260(23):12866-12874

Kunkel TA (1992) DNA replication fidelity. J Biol Chem 267(26):18251-18254

Kunkel TA, Sabatino RD, Bambara RA (1987) Exonucleolytic proofreading by calf thymus DNA polymerase delta. Proc Natl Acad Sci U S A 84(14):4865-4869

Larsson NG (2011) Somatic mitochondrial DNA mutations in mammalian aging. Annu Rev Biochem 79:683-706

Lee HR, Johnson KA (2006) Fidelity of the human mitochondrial DNA polymerase. J Biol Chem 281(47):36236-36240

Lipsky NG, Pedersen PL (1981) Mitochondrial turnover in animal cells. Half-lives of mitochondria and mitochondrial subfractions of rat liver based on [14C]bicarbonate incorporation. J Biol Chem 256(16):8652-8657

Liu Z, Butow RA (2006) Mitochondrial retrograde signaling. Annu Rev Genet 40:159-185

Marver HS, Collins A, Tschudy DP, Rechcigl M Jr (1966) Delta-aminolevulinic acid synthetase. II. Induction in rat liver. J Biol Chem 241(19):4323-4329

Menzies RA, Gold PH (1971) The turnover of mitochondria in a variety of tissues of young adult and aged rats. J Biol Chem 246(8):2425-2429

Mijaljica D, Prescott M, Devenish RJ (2007) Different fates of mitochondria: alternative ways for degradation? Autophagy 3(1):4-9

Miwa S, Lawless C, von Zglinicki T (2008) Mitochondrial turnover in liver is fast in vivo and is accelerated by dietary restriction: application of a simple dynamic model. Aging Cell 7(6):920-923

Mootha VK, Bunkenborg J, Olsen JV, Hjerrild M, Wisniewski JR, Stahl E, Bolouri MS, Ray HN, Sihag S, Kamal M,
Patterson N, Lander ES, Mann M (2003) Integrated analysis of protein composition, tissue diversity, and gene regulation in mouse mitochondria. Cell 115(5):629-640

Nakatogawa H, Suzuki K, Kamada Y, Ohsumi Y (2009) Dynamics and diversity in autophagy mechanisms: lessons from yeast. Nat Rev Mol Cell Biol 10(7):458-467

Narendra D, Tanaka A, Suen DF, Youle RJ (2008) Parkin is recruited selectively to impaired mitochondria and promotes their autophagy. J Cell Biol 183(5):795-803

Nicholas A, Kraytsberg Y, Guo X, Khrapko K (2009) On the timing and the extent of clonal expansion of mtDNA deletions: evidence from single-molecule PCR. Exp Neurol 218(2):316-319

Nicoletti M, Guerri C, Grisolia S (1977) Turnover of carbamyl phosphate synthase, of other mitochondrial enzymes and of rat tissues. Effect of diet and of thyroidectomy. Eur J Biochem 75(2):583-592

Poovathingal SK, Gruber J, Halliwell B, Gunawan R (2009) Stochastic drift in mitochondrial DNA point mutations: a novel perspective ex silico. PLoS Comput Biol 5(11): e1000572

Twig G, Elorza A, Molina AJ, Mohamed H, Wikstrom JD, Walzer G, Stiles L, Haigh SE, Katz S, Las G, Alroy J, Wu M, Py BF, Yuan J, Deeney JT, Corkey BE, Shirihai OS (2008) Fission and selective fusion govern mitochondrial segregation and elimination by autophagy. EMBO J 27(2): 433-446

Vermulst M, Bielas JH, Kujoth GC, Ladiges WC, Rabinovitch PS, Prolla TA, Loeb LA (2007) Mitochondrial point mutations do not limit the natural lifespan of mice. Nat Genet 39(4):540-543

Wallace R, Knecht E, Grisolia S (1986) Turnover of rat liver ornithine transcarbamylase. FEBS Lett 208(2):427-430

Wiesner RJ, Zsurka G, Kunz WS (2006) Mitochondrial DNA damage and the aging process: facts and imaginations. Free Radic Res 40(12):1284-1294

Zhang D, Mott JL, Chang SW, Denniger G, Feng Z, Zassenhaus HP (2000) Construction of transgenic mice with tissuespecific acceleration of Mitochondrial DNA mutagenesis. Genomics 69:151-161 